\title{
Comparative sensitivity and resistance of some strains of Pseudomonas aeruginosa and Pseudomonas stutzeri to antibacterial agents
}

\author{
A. D. RUSSELL AND A. P. MILLS \\ From the Welsh School of Pharmacy, University of Wales Institute of Science and Technology, Cardiff
}

SYNOPSIS A comparison has been made of the sensitivities to various antibiotic and non-antibiotic substances of some strains of Pseudomonas aeruginosa and $P$. stutzeri, the latter including strains isolated from eye and other cosmetic products and from other sources. Whereas $P$. aeruginosa strains showed a high resistance to cetrimide and to benzalkonium chloride, the $P$. stutzeri strains were generally more sensitive to these and to chlorhexidine. The $P$. stutzeri strains were also more sensitive to the various antibiotics tested. The loss of the ability to transfer an $\mathbf{R}$ factor by two strains of $P$. aeruginosa caused no significant change in their drug sensitivity pattern.

The resistance of Pseudomonas aeruginosa to many antibacterial agents has long been a problem (see Brown, 1971, for a comprehensive review). The comparative resistance of other pseudomonads has, in contrast, been little studied. $P$. stutzeri has been reported to be a frequent contaminant of eye cosmetics (Bruch, 1971) and it was thus decided to compare the sensitivity to various substances of some NCTC and ATCC strains and of some strains isolated from eye cosmetics and other sources with some previously studied $P$. aeruginosa strains (Russell, 1972a) and with some recent hospital isolates of the latter organism.

\section{Materials and Methods}

SUBSTANCES USED

These were chlorhexidine diacetate, cetrimide, benzalkonium chloride (BZK), tobramycin, gentamicin, polymyxin B sulphate, ampicillin, carbenicillin, cephaloridine and a new cephalosporin, cephacetrile (the sodium salt of 7-cyanacetamidocephalosporanic acid).

\section{ORGANISMS}

Hospital isolates of $\boldsymbol{P}$. aeruginosa, obtained from hospitals in Cardiff and Dublin, werekindly provided by Dr D. J. Stickler. Five NCTC strains of $P$. aeruginosa $(10662,6750,8203,10701$, and 10490) and two strains bearing $R$ factors $\left(R^{+}+1822\right.$ and $\left.R+3425\right)$, kindly supplied by Dr E. J. L. Lowbury, were also used. Received for publication 4 March 1974.
Three NCTC strains of $P$. stutzeri (10450, 10473 and 10475), two ATCC strains, and some strains isolated from eye and other cosmetic products and from other sources were also studies. The $P$. stutzeri strains are listed in table I. All strains were grown overnight at $37^{\circ} \mathrm{C}$ in $10 \mathrm{ml}$ of nutrient broth (Oxoid no. 2) in McCartney bottles.

\begin{tabular}{ll}
\hline P. stutzeri Strain & Source \\
\hline $10450,10473,10475$ & NCTC \\
17592,17685 & ATCC \\
$1159^{1}$ & Treatment room floor \\
$1195^{1}$ & Scalp wound \\
$1290^{1}$ & Patient's urine \\
$2771^{1}$ & Sink drain \\
$3197^{1}$ & Cutting oil \\
$3282^{1}$ & Sink drain \\
$1512^{2}$ & Brown cosmetic colour material \\
$1514^{2}$ & Eye shadow \\
$1520^{2}$ & Eye shadow \\
$1527^{2}$ & Lernon lotion \\
$1531^{2}$ & Body cream \\
\hline
\end{tabular}

Table I Source of P. stutzeri strains

'Obtained from Dr Marcia A. Moody, Baltimore, Maryland, USA 'Obtained from Dr C. W. Bruch and Dr J. R. Evans, Washington, DC, USA

DETERMINATION OF MINIMUM INHIBITORY CONCENTRATIONS (MICS)

Overnight cultures of the organisms were diluted $1 / 100$ in nutrient broth in McCartney bottles, and these were incubated for two hours at $37^{\circ} \mathrm{C}$ to allow the organisms to be in the logarithmic growth phase. Volumes, each of $0.05 \mathrm{ml}$ (containing about $10^{6}$ 
viable cells) were then dropped on to the surface of nutrient agar (Oxoid) plates containing the desired concentration of the required drug, the plates having previously been dried for three hours at $37^{\circ} \mathrm{C}$. Plates were incubated for 24 hours at $37^{\circ} \mathrm{C}$, and the lowest concentration of a drug which prevented colony formation was taken as the MIC.

\section{Results and Discussion}

The MICs of non-antibiotic substances against the various strains are listed in table II. The hospital isolates of $\boldsymbol{P}$. aeruginosa show a similar response to the NCTC strains, including NCTC 10662 which is used as a 'standard' strain (National Collection of Type Cultures, 1972). Minimum inhibitory concentration values may be slightly different from those described earlier (Russell, 1972a) because of a slight modification in technique. The rather high resistance of $\boldsymbol{P}$. aeruginosa to cetrimide and to BZK is to be noted, although three $P$. stutzeri strains (1290, 2771 , and 3197) are much less susceptible to these agents than the other strains. Whereas most of the $P$. stutzeri strains are inhibited by $<10 \mu \mathrm{g} / \mathrm{ml}$ of chlorhexidine, a few strains are only inhibited at levels corresponding to the MIC values of this compound against $\boldsymbol{P}$. aeruginosa.

Table III gives the MICs of some antibiotics against the various strains. As before, $P$. aeruginosa $\stackrel{\text { F }}{\overrightarrow{0}}$ (NCTC 10662) is used as a 'standard' strain. All strains of $P$. aeruginosa grew in agar containing $\underset{\vec{D}}{\vec{P}}$ $500 \mu \mathrm{g} / \mathrm{ml}$ of cephaloridine, ampicillin, and a newo cephalosporin, cephacetrile (Russell, 1972b). Strain NCTC 10701 is extremely sensitive to carbenicillin $\frac{\overline{\bar{c}}}{\overline{\frac{1}{2}}}$ and is the test organism in its assay. Strains of $\underset{Q}{2}$ $P$. aeruginosa were fairly sensitive to tobramycin, ® polymyxin, and gentamicin, although no allowance was made for the magnesium content of the agar $\vec{\circ}$ medium (Garrod and Waterworth, 1969). Most of $\overrightarrow{\vec{\omega}}$ the $P$. stutzeri strains were inhibited by 20 to $25 \stackrel{\omega}{\omega}$ $\mu \mathrm{g} / \mathrm{ml}$ of carbenicillin and were highly sensitive to polymyxin, tobramycin, and gentamicin. They were generally fairly sensitive to cephaloridine and to ampicillin, but were generally not inhibited even by high concentrations of cephacetrile; in this context, $\stackrel{\vec{\omega}}{ }$ however, two strains (1159 and 1520) were ab-을 normally sensitive to carbenicillin, ampicillin, $\underset{-}{-}$ cephacetrile, and cephaloridine and two other strains of $P$. stutzeri (1195 and 3282) showed an above-average resistance to carbenicillin, ampicillin, $\vec{D}$ and cephaloridine. The possibility that the presence $\vec{v}$ or absence of a $\beta$-lactamase is involved has yet to be ${ }^{+}$ considered.

Moody, Young, and Kenton (1972) have found that $P$. stutzeri strains were susceptible to most of the antibiotics which they tested.

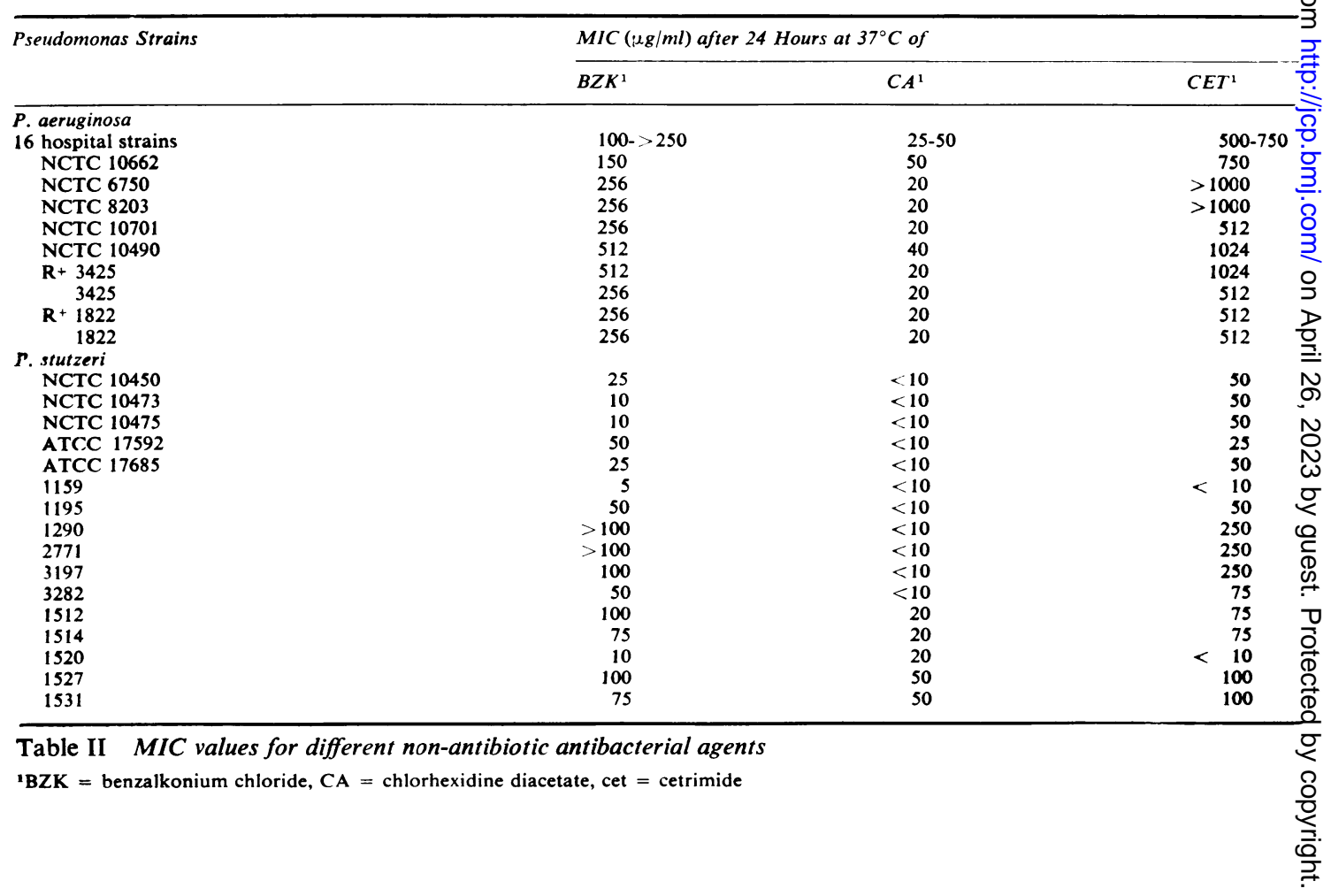




\begin{tabular}{|c|c|c|c|c|c|c|c|}
\hline \multirow[t]{2}{*}{ Pseudomonas Strains } & \multicolumn{7}{|c|}{ MIC $(\mu \mathrm{g} / \mathrm{ml})$ after 24 Hours at $37^{\circ} \mathrm{C}$ of } \\
\hline & Carb & $A m p$ & $C A A$ & Cer & $\boldsymbol{P M}$ & $G M$ & $T o b$ \\
\hline $\begin{array}{l}P . \text { aeruginosa } \\
16 \text { Hospital strains } \\
\text { NCTC } 10662 \\
\text { NCTC } 6750 \\
\text { NCTC } 8203 \\
\text { NCTC } 10701 \\
\text { NCTC } 10490 \\
\text { R+3425 } \\
3425 \\
\mathbf{R}+1822 \\
1822\end{array}$ & $\begin{array}{c}100-250 \\
125 \\
62 \cdot 5 \\
62 \cdot 5 \\
0 \cdot 8 \\
7 \cdot 75 \\
>4000 \\
>4000 \\
>4000 \\
>4000\end{array}$ & $\begin{array}{r}>500 \\
>500 \\
2000 \\
2000 \\
<500 \\
1000 \\
>4000 \\
>4000 \\
>4000 \\
>4000\end{array}$ & $\begin{array}{l}>500 \\
>500 \\
>4000 \\
>4000 \\
>4000 \\
>4000 \\
>4000 \\
>4000 \\
>4000 \\
>4000\end{array}$ & $\begin{array}{r}>500 \\
>500 \\
>4000 \\
4000 \\
2000 \\
4000 \\
>4000 \\
>4000 \\
>4000 \\
>4000\end{array}$ & $\begin{array}{l}5-15 \\
2 \cdot 5-5 \\
32 \\
16 \\
16 \\
16 \\
32 \\
8 \\
32 \\
32\end{array}$ & $\begin{array}{l}1 \cdot 5 \cdot 6 \\
2 \cdot 5 \\
10 \\
2 \cdot 5 \\
2 \cdot 5 \\
10 \\
5 \\
5 \\
10 \\
10\end{array}$ & $\begin{array}{l}2 \\
1 \cdot 5 \\
1 \cdot 5 \\
1 \\
2 \\
5-10 \\
5\end{array}$ \\
\hline $\begin{array}{l}\text { P. stutzeri } \\
\text { NCTC } 10450 \\
\text { NCTC } 10473 \\
\text { NCTC } 10475 \\
\text { ATCC } 17592 \\
\text { ATCC } 17685 \\
1159 \\
1195 \\
1290 \\
2771 \\
3197 \\
3282 \\
1512 \\
1514 \\
1520 \\
1527 \\
1531\end{array}$ & $\begin{array}{c}5 \\
250 \\
50 \\
25 \\
25 \\
<0.5 \\
>40 \\
5 \\
2.5 \\
10 \\
>40 \\
20 \\
20 \\
<0.5 \\
20 \\
20\end{array}$ & $\begin{array}{r}5 \\
25 \\
5 \\
25 \\
25 \\
1 \\
>40 \\
25 \\
25 \\
5 \\
>40 \\
25 \\
10 \\
1 \\
25 \\
10\end{array}$ & $\begin{array}{r}>500 \\
>500 \\
250 \\
>250 \\
15 \\
>500 \\
100 \\
>500 \\
>500 \\
>500 \\
>500 \\
>500 \\
15 \\
>500 \\
>500\end{array}$ & $\begin{array}{c}25 \\
>25 \\
25 \\
10 \\
25 \\
10 \\
100 \\
25 \\
50 \\
10 \\
100 \\
100 \\
2 \cdot 5 \\
5 \\
25 \\
25\end{array}$ & $\begin{array}{l}<2 \cdot 5 \\
<2 \cdot 5 \\
<2 \cdot 5 \\
1 \cdot 25 \\
1 \cdot 25 \\
2 \cdot 5 \\
2 \cdot 5 \\
5 \\
5 \\
2 \cdot 5 \\
2 \cdot 5 \\
5 \\
2 \cdot 5 \\
2 \cdot 5 \\
2 \cdot 5 \\
5\end{array}$ & $\begin{array}{c}<1.5 \\
<1.5 \\
<1.5 \\
1 \\
2 \\
<0.5 \\
1.5 \\
1.5 \\
1.5 \\
1.5 \\
1.5 \\
1.5 \\
5 \\
<0.5 \\
1.5 \\
1.5\end{array}$ & $\begin{array}{l}0 \cdot 5 \\
1 \cdot 5 \\
1 \\
1 \\
1 \\
1 \cdot 5 \\
1 \\
<0 \cdot 5 \\
1 \\
1\end{array}$ \\
\hline
\end{tabular}

Table III MIC values for different antibiotics

Carb $=$ carbenicillin, $\mathbf{A m p}=$ ampicillin, $\mathbf{C A A}=$ cephacetrile, $\mathrm{Cer}=$ cephaloridine, $\mathbf{P M}=$ polymyxin $\mathbf{B}$ sulphate, $\mathbf{G m}=\mathbf{g e n t a m i c i n}$, Tob $=$ tobramycin.

Old subcultures, stored on nutrient agar slopes at $4^{\circ} \mathrm{C}$, of $P$. aeruginosa $\mathrm{R}+1822$ and $\mathrm{R}+3425$ appeared to have lost their ability to transfer their $\mathrm{R}$ factor to an $E$. coli acceptor strain, and accordingly a brief study was made of the sensitivity of these latter strains (designated simply 1822 and 3425) to some of the antibacterial agents. The results (tables II and III) indicate that 3425 and 1822 show a similar sensitivity to antibiotic drugs and to nonantibiotic antibacterial agents as do $R+3425$ and $R+1822$, respectively. In particular, the very high resistance of all four strains to the $4 \beta$-lactam antibiotics is to be noted. Preliminary experiments suggest that, although 3425 and 1822 have lost the ability to transfer the $\mathrm{R}$-factor, they still produce $\beta$-lactamase, and this could obviously contribute to their resistance to these drugs.

Of the pseudomonads (reviewed by Stanier, Palleroni, and Doudoroff, 1966), P. stutzeri NCIB 9040 (NCTC 10473) was found by Wilkinson (1967, 1970 ) to be even more sensitive than $P$. aeruginosa to the chelating agent, ethylenediamine tetraacetic acid (EDTA), and, with $P$. syncyanea, to be reremarkably sensitive to mechanical disruption (Wilkinson, 1970). Differences in cell wall chemistry (Wilkinson and Carby, 1971) and, more especially in the arrangement of wall layers, could be re- sponsible for the comparative sensitivity of $P$. stutzeri to EDTA and to the compounds contained in table I. Both $P$. stutzeri and $P$. syncyanea contain less wall muramic acid than other strains of pseudomonads, and appear to stand apart from other species and from each other with regard to their cell wall structure according to Wilkinson (1970), who also suggests that the chemical basis for sensitivity of $P$. stutzeri to EDTA may not be strictly comparable with that of the other Pseudomonas sp investigated. However, most of the strains of $\boldsymbol{P}$. stutzeri are sensitive to ampicillin and are more sensitive to carbenicillin than the hospital isolates of $P$. aeruginosa (table II).

The pathogenicity of $P$. stutzeri strains is not clear (Bruch, 1971). However, their occurrence as contaminants in eye and other cosmetics and in other sources suggests that more knowledge is needed of their sensitivity to antimicrobial agents. The results presented here indicate that they are generally more sensitive than $\boldsymbol{P}$. aeruginosa to various types of antibiotics, and to non-antibiotic antibacterial agents of the type used as disinfectants and/or as preservatives in a wide variety of pharmaceutical and cosmetic products.

We thank Miss L. M. Smart for technical assistance 
and the Wellcome Trust for a research studentship to one of us (A.P.M.) We are also grateful to Dr C. W. Bruch and Dr J. R. Evans of the Drug Microbiology Branch, FDA, Washington, DC, for allowing us to study $P$. stutzeri strains isolated from eye and other cosmetic products, and to $\mathrm{Dr}$ Marcia A. Moody of the National Cancer Institute, Baltimore, Maryland, for allowing us to study strains isolated from other sources.

\section{References}

Brown, M. R. W. (1971). Inhibition and destruction of Pseudomonas aeruginosa. In Inhibition and Destruction of the Microbial Cell, edited by W. B. Hugo, pp. 307-367. Academic Press, London.

Bruch, C. W. (1971). Cosmetic sterility vs. microbial control. Amer. Perfumer Cosmet. 86, 182.

Garrod, L. P., and Waterworth, P. M. (1969). Effect of medium composition on the apparent sensitivity of Pseudomonas aeruginosa to gentamicin. J. clin. Path., 22, 534-538.

Moody, M. R., Young, V. M., and Kenton, D. M. (1972). In vitro antibiotic susceptibility of Pseudomonads other than Pseudo- $\overrightarrow{\bar{F}}$ monas aeruginosa recovered from cancer patients. Antimicrob. $\stackrel{\oplus}{+}$ Agents and Chemother., 2, 344-349.

National Collection of Type Cultures (1972). Catalogue of the National Collection of Type Cultures, 5 th ed. HMSO, London.

Russell, A. D. (1972a). Resistance of $\mathrm{R}^{+}$and other strains of Pseudo- $\frac{\mathcal{D}}{}$ monas aeruginosa to non-antibiotic antibacterial agents. $(\mathbb{D}$ Lancet, $2,332$.

Russell, A. D. (1972b). In vitro studies with Ciba 36, 278-Ba, a new cephalosporin derivative. Microbios, 6, 221-230.

Stanier, R. Y., Palleroni, N. J., and Doudoroff, M. (1966). The $\overrightarrow{0}$ aerobic pseudomonads: a taxomonomic study. J. gen. Microbiol., 43, 159-271.

Wilkinson, S. G. (1967). The sensitivity of pseudomonads to ethylenediamine tetraacetic acid. J. gen. Microbiol., 47, 67-76.

Wilkinson, S. G. (1970). Cell walls of Pseudomonas species sensitive to ethylenediamine tetraacetic acid. J. Bact., 104, 1035-1044.

Wilkinson, S. G., and Carby, K. A. (1971). Amino sugars in the cell N walls of Pseudomonas species. J. gen. Microbiol., 661, 221-227. 